\title{
A Study of the Usefulness of Pediatric Balance Scale as a Prediction Indicator for Gross Motor Function Classification System in Children with Cerebral Palsy
}

\author{
Hyoung-Won Lim \\ Department of Physical Therapy College of Health Sciences Dankook University, Cheonan, Chungcheongnam-do, Korea
}

\begin{abstract}
Purpose: The purpose of this study was to evaluate the relation between PBS scores and GMFCS levels and to examine whether pediatric balance scale (PBS) scores were useful for predicting gross motor functional classification system (GMFCS) levels in children with cerebral palsy.

Methods: This cross-sectional study was performed conducted for to evaluatione of PBS and GMFCS using in 26 children with cerebral palsy (16 males and 10 females with GMFCS level I to III). PBS total and item scores at different levels of GMFCS were measured.

Results: The hHigh PBS item average scores obtained from standing and postural change dimensions except sitting dimension were observed at the low levels of GMFCS and these results were statistically significant $(p<0.05)$. The relation between PBS (standing and postural change dimensions) and GMFCS levels were was significantly different, except the relation between PBS sitting dimension and GMFCS levels showing a ceiling effect.

Conclusion: GMFCS is designed to for classificationy of gross motor functions emphasizing on walking movement and PBS is was developed to for evaluatione of functional balance. Based on the results of this study showing high relation between GMFCS levels and PBS scores, PBS scores can be used for predicting GMFCS levels.
\end{abstract}

Keywords: Cerebral palsy, Pediatric balance scale, Gross motor function classification system

\section{서 론}

뇌성마비 아동은 손상된 근육 긴장도와 비정상적인 자세조절로 인 하여 기능적인 균형 소실을 초래한다.1.2 자세조절은 신체분절의 기저 면과 정렬의 한계 내에서 질량중심(center of mass)을 유지하는 능력이 요구된다.,34 뇌성마비에서 자세조절의 소실은 변경된 신경근육 반응 을 결정하는 감각처리와 생체역학적 정렬의 변경을 초래한다. ${ }^{5}$ 균형 과 정립 자세조절은 움직임의 기본 구성 요소이며, 기능적인 균형은 대동작 능력의 요소로 정의되며, 빈약한 균형은 일상생활동작에 포 함되는 기능적인 과제에 곤란을 야기한다. ${ }^{6}$

소아 물리치료사들은 대동작 운동기능을 향상시키기 위해 치료 세션에서 자주 균형훈련을 포함시킨다. ${ }^{78}$ 이러한 균형은 기능적인 활 동과 관련되기 때문에 신뢰할 수 있고 간단한 기능적인 균형측정은 물리치료 중재의 효과를 평가하기 위해 필요하다. 뇌성마비 아동에
서 균형은 전통적으로 대동작 운동기능(Gross Motor Function Measure, GMFM)과 실험실 같이 표준화된 개발평가도구들을 통해 평가 됐다.9-11 소아균형검사(Pediatric Balance Scale, PBS)는 신경운동 장애 가 있는 아동의 기능적인 균형을 평가하기 위해 신뢰할 수 있는 도구 이며, 그리고 이러한 아동들이 효과적으로 일상생활 수행이 가능하 도록 하는 자세조절의 요소를 평가하는 도구이다.12,13 선행연구에서 $\mathrm{PBS}$ 는 뇌성마비에서 GMFM 총점, 보행속도, 그리고 앉았다 일어서 기 검사결과에서 높은 상관관계를 보였다. ${ }^{5}$ 또한 모든 PBS 점수는 대 동작 운동기능에 강한 예측인자이며, 일상생활 활동에 좋은 예측인 자이며, 특별히 추적관찰에서 이동성 도메인에 좋은 예측인자로 보 고되었다. ${ }^{5} \mathrm{GMFM}$ 은 운동능력을 평가하기 위하여 널리 사용되며 검 증된 도구이다. 그러나, 완전히 기능적인 균형능력을 평가할 수는 없 다. ${ }^{14}$ 전신운동 기능 분류시스템(Gross Motor Function Classification System, GMFCS)은 움직임의 질을 판단하지 않고 앉기와 걷기 움직임
Received Jan 13, 2016 Revised Feb 18, 2016

Accepted Feb 22, 2016

Corresponding author Hyoung-Won Lim

E-mail movt12@hanmail.net
Copylight (C) 2016 The Korea Society of Physical Therapy

This is an Open Access article distribute under the terms of the Creative Commons Attribution Non-commercial License (Http:// creativecommons.org/license/by-nc/3.o.) which permits unrestricted non-commercial use, distribution, and reproduction in any medium, provided the original work is properly cited. 
에 중점을 두고 대동작 운동기능을 분류하여 뇌성마비 아동에서 신 경운동 손상 정도를 평가하기 위해 개발되었다. ${ }^{15}$

뇌성마비 아동의 연구에서 $\mathrm{PBS}$ 의 타당도를 보고한 논문과 균형을 평가하기 위한 논문이 대부분이며 $\mathrm{PBS}$ 와 다른 평가도구들과의 상관 관계를 분석한 연구는 부족한 실정이다. ${ }^{14-18}$ 신뢰할 수 있는 도구를 이용하여 자세조절을 평가한다면 자세조절로 인한 기능적인 손상의 더 나은 이해를 제공할 수 있을 것이다. 이에 본 연구에서는 뇌성마비 아동의 서로 다른 수준의 GMFCS에서 PBS 총점과 영역별 점수를 확 인하고, PBS 점수를 이용하여 GMFCS 수준의 예측인자로 사용될 수 있는지 여부를 평가하고자 한다.

\section{연구방법}

\section{1. 연구대상}

본 연구는 인천과 청주의 재활병원과 복지관에서 외래로 치료를 받 고 있는 뇌성마비 아동 26 명을 대상으로 실시하였으며, 실험에 참여 한 아동 및 보호자에게 연구의 목적과 실험 방법에 대하여 설명하고 동의를 구하였다. 연구 대상자의 선정 조건은 첫째 뇌성마비로 진단 받은 아동, 둘째 독립적으로 4 초 이상 선 자세의 유지가 가능한 아동, 전신운동 기능 분류시스템(gross motor function classification system, GMFCS) 평가상 I-III 단계에 속하고, 연구자가 지시하는 내용을 이해 하고 협조할 수 있는 아동으로 하였다.

\section{2. 실험방법 \\ 1) 측정도구 \\ (1) 소아균형검사}

표준화된 기립 균형 측정도구로 경도에서 중등도의 운동 손상이 있 는 학령기(School-aged children) 뇌성마비 아동들의 기능적인 균형 평 가에 사용되며, 검사-재검사 방법에서 급간내 상관 계수는 0.99 로 신 뢰도가 확인된 평가 도구이다.16 검사는 15 분 안에 측정이 가능하며, 전문화된 장비의 사용을 요구하지 않는다. $\mathrm{PBS}$ 를 구성하는 14 개 항 목은 앉기, 서기, 자세변화의 총 3 가지 영역으로 구성되며, 이것은 아 동이 안전하게 수행해야 하고 가정, 학교, 지역사회 내에서 독립적인 기능을 수행해야 하는 많은 기능적인 활동을 평가한다. 본 연구에서 는 PBS 앉기 영역(5항목), 서기 영역(4, 6, 7, 8, 9, 11, 12, 14항목), 자세변 화 영역 $(1,2,3,10,13$ 항목)으로 설정하여 각 GMFCS 레벨에 따른 PBS 영역별 점수를 비교하였다. 각 항목은 0 점에서 4 점까지 점수가 매겨 지며, 높은 점수일수록 더 나은 균형능력을 의미한다.

\section{(2) 전신운동 기능 분류시스템}

GMFCS는 뇌성마비 아동의 기능적인 분류를 나타내며, 대동작 운동
기능 활동을 수행하는 능력과 제한을 기초로 하여 아동의 수준을 결정하는 것을 목적으로 한다. 아동들은 다음과 같이 다섯 단계로 분류할 수 있다. 레벨 I은 제한 없이 걷는 능력을 나타내며, 레벨 II는 제한이 있는 걷는 능력을 나타내며, 레벨 III는 휴대용 이동 도구를 사용하여 걷는 아동으로 구성되며, 레벨 IV는 자가 이동성에 제한을 보이는 아동을 포함하며, 레벨 $\mathrm{V}$ 는 수동휠체어로 이동되는 아동으 로 이루어졌다. ${ }^{19}$

\section{2) 실험절차}

본 연구는 "Correlation between the selective control assessment of lower extremity and pediatric balance scale scores in children with cerebral palsy"의 하위 연구 중 하나이다. ${ }^{20}$ 본 연구에서 PBS는 주요 측정결과 중 하나이며, 따라서 기존에 개발된 도구들과의 관계를 알아보는 것을 통하여 더 나은 기능적인 손상의 이해를 제공하고자 실시하였다.

평가는 소아물리치료 경력 15 년의 치료사 1 명이 평가하여 점수를 부여하였다. 본 연구에서는 PBS의 각 항목마다 3 회 측정하여 가장 좋 은 점수를 선택하였다. 본 연구에서 아동들은 GMFCS에 따라 분류 하였으며, GMFCS level I-III 아동을 대상으로 하였다.

\section{3) 자료분석}

연구대상자의 일반적인 특성은 빈도분석을 실시하였고, GMFCS level 에 따른 PBS 서기 항목, 앉기 항목과 자세변화 항목의 평균점수를 비 교하기 위하여 일원배치 분산분석(One-way ANOVA)을 실시하였다. 각 항목별 집단 간 평균점수에 차이가 있는지를 알아보기 위한 사후 검정은 least significant difference (LSD) 분석방법으로 하였다. 또한 GMFCS level과 PBS 점수와의 관련성을 분석하기 위하여 다중회귀분 석(multiple regression analysis)을 실시하였으며, GMFCS level을 가변 수로 치환하고 입력방법으로 분석하였다. 수집된 자료는 SPSS ver 21.0 을 이용하여 통계 분석하였다. 유의수준 $\alpha=0.05$ 로 하였다.

\section{결 과}

\section{1. 연구대상자의 일반적 특성}

연구대상자의 일반적 특성은 다음과 같다. 성별분포는 남자가 $61.5 \%$ 로 여자 $38.5 \%$ 보다 더 많았고, 대상자의 평균연령은 $9.1 \pm 2.2$ 세였고, 평균신장은 $124.5 \pm 14.7 \mathrm{~cm}$ 였다. 뇌성마비아동(cerebral palsy)의 유형 은 모든 level에서 경직형 양하지마비(spastic diplegia)가 전체의 $69.2 \%$ 로 가장 많은 빈도를 보였다(Table 1).

\section{GMFCS level에 따른 PBS 항목의 평균점수}

GMFCS level에 따른 PBS 항목의 평균점수를 분석한 결과 서기영역에 
서 level I의 점수는 $3.73 \pm 0.21$ 점, level II의 점수는 $2.69 \pm 0.73$ 점이었으 며, level III의 점수는 $2.38 \pm 0.13$ 점으로 level I에서 가장 점수가 높았고 통계적으로 유의하였다 $(\mathrm{p}<0.00)$. GMFCS level 에 따른 앉기 영역의 PBS점수는 level I과 II, III의 점수가 모두 동일하게 나타나 유의한 차 이를 보이지 않았다. GMFCS level 에 따른 자세변화 영역에서 level I의 점수는 $3.89 \pm 0.16$ 점이었고, level II의 점수는 $3.12 \pm 0.78$ 점이었으며, level III는 $2.60 \pm 0.72$ 점으로 level I에서 점수가 가장 높았고 통계적으로 유의하였다( $\mathrm{p}<0.01)$. GMFCS level 에 따른 PBS 총점의 경우에도 level I 의 점수는 $3.81 \pm 0.14$ 점으로 level II의 $2.94 \pm 0.68$ 점과 level III의 $2.57 \pm$ 0.31 점 보다 높게 나타나 유의한 차이를 보였다 $(\mathrm{p}<0.05)$ (Table 2).

\section{GMFCS level과 PBS 서기 영역 간의 다중회귀분석 결과}

GMFCS level과 PBS 서기영역 간의 관련성을 분석하기 위하여 GMFCS level 항목을 가변수로(dummy variable)로 치환하고 회귀분석을 실 시한 결과는 Table 3과 같다. GMFCS level I을 기준으로 했을 때 level II 의 경우는 PBS점수가 평균 1.043 점 감소하였고, level III에서는 평균 1.356 점 감소되어 level이 높은 경우 PBS 점수가 더 낮았고 통계적으로 유의하였다. 이 회귀식에서 GMFCS level이 PBS 서기 영역에 대해 갖 는 변수의 설명력은 $60.9 \%$ 였다(Table 3 ).

Table 1. General characteristics of subjects

\begin{tabular}{lcccc}
\hline \multirow{2}{*}{ Characteristics } & \multicolumn{3}{c}{ GMFCS Level } & \multirow{2}{*}{ Total } \\
\cline { 2 - 4 } Gender & I & II & III & \\
\cline { 2 - 4 } Male & $8(61.5)^{*}$ & $7(70.0)$ & $1(33.3)$ & $16(61.5)$ \\
Female & $5(38.5)$ & $3(30.0)$ & $2(66.7)$ & $10(38.5)$ \\
Age (year) & $9.8 \pm 2.7^{+}$ & $8.2 \pm 1.4$ & $9.3 \pm 0.6$ & $9.1 \pm 2.2$ \\
Height (cm) & $130.4 \pm 16.3$ & $118.7 \pm 9.3$ & $118.3 \pm 16.5$ & $124.5 \pm 14.7$ \\
CP type & & & & \\
Spastic hemiplegia & $5(38.5)$ & $0(0.0)$ & $0(0.0)$ & $5(19.2)$ \\
Spastic diplegia & $8(61.5)$ & $7(70.0)$ & $3(100.0)$ & $18(69.2)$ \\
Ataxia & $0(0.0)$ & $2(20.0)$ & $0(0.0)$ & $2(7.7)$ \\
Athetoid & $0(0.0)$ & $1(10.0)$ & $0(0.0)$ & $1(3.8)$ \\
Total & $13(100.0)$ & $10(100.0)$ & $3(100.0)$ & $26(100.0)$ \\
\hline
\end{tabular}

GMFCS: Gross motor function classification system, CP: Cerebral palsy.

${ }^{*}$ Number(\%); ${ }^{+}$Values are mean \pm SD.

\section{GMFCS level과 PBS 자세변화 영역 간의 다중회귀분석 결과}

GMFCS level과 PBS 자세변화 영역 간의 관련성을 분석하기 위하여 GMFCS level 항목을 가변수로(dummy variable)로 치환하고 회귀분석 을 실시한 결과는 Table 4와 같다. GMFCS level I을 기준으로 했을 때 level II의 경우는 PBS 점수가 평균 0.772점 감소하였고, level III에서는 평균 1.292 점 감소되어 level 높은 경우PBS 점수가 더 낮았고 통계적으 로 유의하였다(p<0.01). 이 회귀식에서 GMFCS level이 PBS 자세변화 영역에 대해 갖는 변수의 설명력은 $46.1 \%$ 였다(Table 4$)$.

\section{GMFCS level과 PBS 총점 간의 다중회귀분석 결과}

GMFCS level과 PBS 총점 간의 관련성을 분석하기 위하여 GMFCS level 항목을 가변수로(dummy variable)로 치환하고 회귀분석을 실시 한 결과는 Table 5 와 같다. GMFCS level I을 기준으로 했을 때 level II의 경우는 PBS 점수가 평균 0.872점 감소하였고, level III에서는 평균 1.236 점 감소되어 level이 높은 경우 PBS 점수가 더 낮았고 통계적으로 유의하였다 $(\mathrm{p}<0.01)$. 이 회귀식에서 GMFCS level이 PBS 총점에 대해 갖는 변수의 설명력은 $57.8 \%$ 였다(Table 5).

\section{고 찰}

뇌성마비에서 주요한 자세 기능부전은 특별히 기능적인 활동 수행

Table 3. Regression analysis between GMFCS level and PBS standing dimension

\begin{tabular}{lcccc}
\hline GMFCS level & $\mathrm{B}$ & $\mathrm{S} . \mathrm{E}$ & $\mathrm{t}$ & $\mathrm{p}$-value \\
\hline Constant & 3.731 & 0.134 & 27.899 & 0.000 \\
Level II & -1.043 & 0.203 & -5.144 & 0.000 \\
Level III & -1.356 & 0.309 & -4.390 & 0.000 \\
\hline
\end{tabular}

Dependent variable: PBS, method: enter, $R^{2}: 0.609$.

Table 4. Regression analysis between GMFCS level and PBS postural change

\begin{tabular}{lcccc}
\hline GMFCS level & $\mathrm{B}$ & $\mathrm{S} . \mathrm{E}$ & $\mathrm{t}$ & $\mathrm{p}$-value \\
\hline Constant & 3.892 & 0.151 & 25.838 & 0.000 \\
Level II & -.772 & 0.228 & -3.380 & 0.003 \\
Level III & -1.292 & 0.348 & -3.715 & 0.001 \\
\hline
\end{tabular}

Dependent variable: PBS, method: enter, $\mathrm{R}^{2}: 0.461$.

Table 2. PBS scores related to GMFCS levels

\begin{tabular}{|c|c|c|c|c|c|c|}
\hline PBS dimension & $\begin{array}{c}\text { Level I } \\
(N=13)\end{array}$ & $\begin{array}{c}\text { Level II } \\
(\mathrm{N}=10)\end{array}$ & $\begin{array}{l}\text { Level III } \\
(N=3)\end{array}$ & Average & $p$ & Post-hoc \\
\hline Standing & $3.73 \pm 0.21 *$ & $2.69 \pm 0.73$ & $2.38 \pm 0.13$ & $3.17 \pm 0.74$ & 0.000 & $A>B, C$ \\
\hline Sitting & 4.00 & 4.00 & 4.00 & 4.00 & - & - \\
\hline Postural change & $3.89 \pm 0.16$ & $3.12 \pm 0.78$ & $2.60 \pm 0.72$ & $3.45 \pm 0.71$ & 0.001 & $A>B, C$ \\
\hline Total & $3.81 \pm 0.14$ & $2.94 \pm 0.68$ & $2.57 \pm 0.31$ & $3.33 \pm 0.66$ & 0.000 & $A>B, C$ \\
\hline
\end{tabular}

PBS: Pediatric balance scale, A: level I, B: level II, C: level III.

*Values are mean \pm SD; ${ }^{*}$ post-hoc, LSD analysis. 
Table 5. Regression analysis between GMFCS level and PBS total scores

\begin{tabular}{lcccc}
\hline GMFCS level & $\mathrm{B}$ & $\mathrm{S} . \mathrm{E}$ & $\mathrm{t}$ & $\mathrm{p}$-value \\
\hline Constant & 3.808 & 0.124 & 30.800 & 0.000 \\
Level II & -0.872 & 0.187 & -4.651 & 0.000 \\
Level III & -1.236 & 0.286 & -4.330 & 0.000
\end{tabular}

Dependent variable: $\mathrm{PBS}$, method: enter, $\mathrm{R}^{2}: 0.578$.

시, 바른 순서로 자세근육의 활성을 협응할 수 없는 것이다.,21 이것은 비정상적인 근육동원 패턴, 높은 동시수축, 그리고 변경된 근육동원 순서를 초래할 수 있다.22 일상생활동작에 포함되는 기능적인 과제 수 행의 곤란은 움직임의 기본 구성요소인 균형과 정립 자세조절이 불 충분할 때 야기될 수 있다. ${ }^{8.23}$ 서기 활동을 실행하는 동안 안정성을 유지하는 능력인 기능적인 균형은 PBS로 측정할 수 있다. ${ }^{16}$ 소아 물리 치료 영역에서, 신뢰도와 타당도가 높은 측정 도구는 치료 계획 및 측 정 결과의 설정을 위한 확실한 정보를 제공할 수 있다. ${ }^{24}$ 따라서 기존 에 개발된 도구들과의 관계를 알아보는 것을 통한 신뢰도와 타당도 검증이 필요하다.

본 연구는 뇌성마비 아동에서 서로 다른 수준의 GMFCS에서 PBS 총점과 영역별 점수를 확인하고, $\mathrm{PBS}$ 점수를 이용하여 GMFCS 수준 의 예측인자로 사용될 수 있는지 여부를 평가하고자 하였다. 본 연구 결과에서 GMFCS에 따른 PBS 항목의 평균점수는 앉기 영역을 제외 한 서기 영역과 자세변화 영역에서 레벨이 낮을수록 높게 나타났으 며 통계적으로 유의한 차이를 보였다( $\mathrm{p}<0.05)$. 반면 GMFCS level에 따른 PBS 앉기 영역 점수에서는 level III로 분류된 아동들도 도움 없 이 앉을 수 있기 때문에 유의한 차이가 없었다. Yi 등는 $\mathrm{PBS}$ 총점이 운동능력(GMFM D and E 영역 점수)과 밀접한 상관관계를 가지며, 서기 및 걷기와 관련된 균형 기능을 검사할 수 있다고 하였다. 본 연구 에서 이용한 GMFCS는 앉기와 걷기에 중점을 두고 대동작 운동기능 을 분류한 것이므로 레벨이 낮을수록(level I) 서기 영역과 자세변화 영역에서 점수가 높게 나타난 본 연구결과와 일치한다고 할 수 있다.

본 연구에서는 GMFCS level과 PBS 서기 영역 간의 높은 관련성을 나타냈으며, 이러한 결과는 GMFCS level II 그룹보다 I 그룹에서 더 좋 은 균형과 더 독립적으로 움직일 수 있기 때문이며, 이것은 한발로 서 기, 일자보행(tandem)에서 도움 없이 서기, 공간에서 자신의 신체를 돌리기와 같은 과제를 수행할 때 쉽게 할 수 있어 점수 차이를 보인다 고 하였다. 또한 GMFCS level II \& III 그룹 간의 PBS 점수 차이는 독립 적인 실내 보행과 관련된다고 하였다. 본 연구에서도 그룹 II와 III 그 룹 간의 점수 차이 역시 같은 서기 항목에서 관찰되었다. 상기의 과제 들은 정적 및 동적 자세조절을 필요로 하며, ${ }^{19}$ level III로 분류된 아동 에서는 실내에서 조차도 보조 이동도구 없이는 걸을 수 없기 때문에 서기 영역의 과제 수행 시 그룹 II로 분류된 아동보다 더욱 문제에 직 면할수 있다. ${ }^{25}$
GMFCS level과 PBS 자세변화 영역의 관련성 역시 레벨이 높을수 록 PBS 점수가 더 낮았으며 통계적으로 유의하였다 $(\mathrm{p}<0.01)$. 자세변 화 영역의 과제들은 (예, 앉은 자세에서 서기 그리고 선 자세에서 앉기 등) 동심성-편심성(concentric-eccentric) 대퇴사두근의 조절이 요구되 는 과제들로 신경운동 손상을 가진 뇌성마비에서는 수행하기 어려 운 과제라고 하였다. ${ }^{26}$ 이러한 이유로 본 연구에서도 GMFCS level 간 의 점수 차이가 보였다고 생각된다. Palisano 등 ${ }^{19}$ 은 level I에만 있는 균 형능력과 독립적으로 움직이는 능력이 GMFCS 레벨 I과 II를 구별하 는 주요한 운동과제라고 하였다.

안정된 자세는 넘어짐을 방지하고 움직임을 안전하게 수행할 수 있 게 하며, 이것은 균형기능을 향상시켜 독립적으로 걸을 수 있는 능력 을 증진시킨다. ${ }^{3} \mathrm{GMFCS}$ II로 분류된 경직형 뇌성마비 아동에서 자세 조절을 평가한 다른 연구에서는 아동들이 선 자세에서 팔 뻗기 (reaching) 동작에 앞서 선행적인 조정(anticipatory adjustment)의 사용 이 나타난다고 하였으나, 높은 근활성도의 변동성을 갖는다고 하였 다. ${ }^{25}$ 동적균형은 보행기능과 밀접한 관련성이 있으며, 보행분석에서 불량한 신체정렬 및 지절간 협응 그리고 느린 보행에서는 보행 시 압 력중심(center of pressure, $\mathrm{CoP}$ ) 변위가 크다고 보고하였다.' 이러한 이 유로 균형능력과 독립적으로 움직이는 능력이 더 좋은 GMFCS level I 그룹이 II와 III 그룹보다 기능적인 균형능력 및 자세조절의 요소를 평 가하는 도구인 PBS 검사에서 더 높은 점수를 얻었다고 생각할 수 있 다. 각 GMFCS 레벨에서 제한된 대상자 수에도 불구하고 GMFCS 레 벨 간의 PBS 점수에서 유의한 차이가 있었다. 따라서 본 연구 결과만 을 놓고 볼 때 GMFCS와 PBS와는 높은 관련성이 있다고 해석할 수 있다.

$\mathrm{Lim}^{20}$ 은 하지의 운동손상 정도를 평가할 수 있는 선택적 운동조절 평가와 기능적인 균형 평가에 사용되는 PBS간의 높은 상관관계를 보고하였다. 더 나은 선택적 운동조절 평가는 대동작 기능의 증가와 함께 동시작용(synergy) 패턴을 감소시켜 자세조절 요소인 $\mathrm{PBS}$ 를 개 선시킨다고 하였다. ${ }^{20}$ 그러므로 본 연구에서 신경운동 손상 정도를 평 가하기 위해 개발된 GMFCS와 자세조절 요소를 평가할 수 있는 PBS 와의 높은 관련성은 소아 물리치료에서 치료의 효과를 객관적으로 평가하는데 유용하게 사용될 수 있을 것으로 생각된다.

본 연구의 제한점은 다음과 같다. 먼저 대상자의 수가 적어 연구 결 과를 일반화하는 데 제한이 된다. 또한 실험 대상을 독립적으로 4 초 이상 선 자세의 유지가 가능한 아동으로 하여 GMFCS level I-III만을 포함시켰다는 것이며, 실조형(ataxia)이나 무정위형(athetoid)과 같은 뇌성마비 참가자 수가 적기 때문에 뇌성마비 아동을 유형별로 분석 하지 못하였다는 것이다.

향후 연구에서는 보다 많은 대상자를 통한 연구와 중증의 뇌성마 비 아동을 대상으로 한 연구가 필요할 것으로 생각된다. 또한 뇌성마 
비 유형에 따른 전신운동 기능 분류시스템과 소아균형검사와 관련 성을 분석하는 연구가 이루어져야할 것이다.

\section{REFERENCES}

1. Liao HF, Jeng SF, Lai JS et al. The relation between standing balance and walking function in children with spastic diplegic cerebral palsy. Dev Med Child Neurol. 1997;39(2):106-12.

2. Kang JH, Kim CY, Ohn JM et al. The effect of hinged ankle-foot orthosis on walking function in children with spastic diplegic cerebral palsy: A Cross-Sectional Pilot Study. J Kor Phys Ther. 2015;27(1):43-9.

3. Pavão SL, Barbosa KA, Sato Tde $\mathrm{O}$ et al. Functional balance and gross motor function in children with cerebral palsy. Res Dev Disabil. 2014; 35(10):2278-83

4. Seo HJ, Kim JH, Choi MJ et al. The effects of gluteal taping on pelvic alignment, trunk stability, and balance during sitting in children with unilateral cerebral palsy. J Kor Phys Ther. 2014;26(5):308-14.

5. Gan SM, Tung LC, Tang YH et al. Psychometric properties of functional balance assessment in children with cerebral palsy. Neurorehabil Neural Repair. 2008;22(6):745-53.

6. Liao HF, Hwang AW. Relations of balance function and gross motor ability for children with cerebral palsy. Percept Mot Skills. 2003;96 (3Pt2): 1173-84.

7. Lexell JE, Downham DY. How to assess the reliability of measurements in rehabilitation. Am J Phys Med Rehabil. 2005;84(9):719-23.

8. Shumway-Cook A, Hutchinson S, Kartin D et al. Effect of balance training on recovery of stability in children with cerebral palsy. Dev Med Child Neurol. 2003;45(9):591-602.

9. Rose J, Wolff DR, Jones VK et al. Postural balance in children with cerebral palsy. Dev Med Child Neurol. 2002;44(1):58-63.

10. Cherng RJ, Su FC, Chen JJ et al. Performance of static standing balance in children with spastic diplegic cerebral palsy under altered sensory environments. Am J Phys Med Rehabil. 1999;78 (4):336-43.

11. Liao HF, Mao PJ, Hwang AW. Test-retest reliability of balance tests in children with cerebral palsy. Dev Med Child Neurol. 2001;43(3):180-6.

12. Ries LG, Michaelsen SM, Soares PS et al. Cross-cultural adaptation and reliability analysis of the Brazilian version of Pediatric Balance Scale (PBS). Rev Bras Fisioter. 2012;16(3):205-15.

13. Franjoine MR, Gunther JS, Taylor MJ. Pediatric balance scale: a modi- fied version of the Berg balance scale for the school-age child with mild to moderate motor impairment. Pediatr Phys Ther. 2003;15(2):114-28.

14. Yi SH, Hwang JH, Kim SJ et al. Validity of pediatric balance scales in children with spastic cerebral palsy. Neuropediatrics. 2012;43(6):307-13.

15. Palisano R, Rosenbaum P, Walter $S$ et al. Development and reliability of a system to classify gross motor function in children with cerebral palsy. Dev Med Child Neurol. 1997;39(4):214-23.

16. Woollacott MH, Burtner P, Jensen J et al. Development of postural responses during standing in healthy children and children with spastic diplegia. Neurosci Biobehavl Rev. 1998;22(4):583-9.

17. Ko JY, Kim GW. Test-retest, Inter-rater, and Intra-rater Reliability of a Pediatric Balance Scale in Children with Cerebral Palsy. J Kor Phys Ther. 2010;22(4):43-8.

18. Kim GW, Ko JY, Baek SG . The reliability of a pediatric balance scale based on the raters' clinical work experience and test experience. J Kor Phys Ther. 2010;22(6):35-42.

19. Palisano RJ, Cameron D, Rosenbaum PL et al. Stability of the gross motor function classification system. Dev Med Child Neurol. 2006;48(6): 424-8.

20. Lim HW. Correlation between the selective control assessment of lower extremity and pediatric balance scale scores in children with spastic cerebral palsy. J Phys Ther Sci.2015;27(12):3645-49.

21. Horak FB. Clinical measurement of postural control in adults. Phys Ther. 1987;67(12):1881-5.

22. Corrêa JC, Corrêa FI, Franco RC et al. Corporal oscillation during static biped posture in children with cerebral palsy. Electromyogr Clinl Neurophysiol. 2007;47(3):131-6.

23. Newell KM, Slobounov SM, Slobounova BS et al. Short-term non-stationarity and the development of postural control. Gait Posture. 1997; 6(1):56-62.

24. Kwon TG, Yi SH, Kim TW et al. Relationship between gross motor function and daily functional skill in children with cerebral. Ann Rehabil Med. 2013;37(1):41-9.

25. Pavão SL, dos Santos AN, Woollacott MH et al. Assessment of postural control in children with cerebral palsy: a review. Res Dev Disabil. 2013; 34(5):1367-75.

26. Woollacott MH, Shumway-Cook A. Postural dysfunction during standing and walking in children with cerebral palsy: What are the underlying problems and what the new therapies might improve balance? Neural Plast. 2005;12(2-3):211-9. 